\title{
Injectable laminin-functionalized hydrogel for nucleus pulposus regeneration
}

\author{
Aubrey T. Francisco ${ }^{a}$, Robert J. Mancino ${ }^{\mathrm{a}}$, Robby D. Bowles ${ }^{\mathrm{a}}$, Jonathan M. Brunger ${ }^{\mathrm{a}, \mathrm{b}}$, \\ David M. Tainter ${ }^{c}$, Yi-Te Chen ${ }^{\text {b,d,e,f }}$, William J. Richardson ${ }^{b}$, Farshid Guilak ${ }^{\mathrm{b}, \mathrm{a}}$, \\ Lori A. Setton ${ }^{a, b, *}$
}

a Department of Biomedical Engineering, Duke University, Durham, NC, USA

b Department of Orthopaedic Surgery, Duke University Medical Center, Durham, NC, USA

${ }^{\mathrm{c}}$ Duke - NUS Graduate Medical School, Singapore

${ }^{\mathrm{d}}$ Department of Orthopedics, Shin Kong Wu Ho-Su Memorial Hospital, Taipei City, Taiwan

e School of Medicine, Fu-Jen Catholic University, New Taipei City, Taiwan

${ }^{\mathrm{f}}$ Department of Orthopedics, National Yang-Ming University, Taipei City, Taiwan

\section{A R T I C L E I N F O}

\section{Article history:}

Received 5 June 2013

Accepted 23 June 2013

Available online 10 July 2013

\section{Keywords:}

Laminin

Nucleus pulposus

Intervertebral disc

Polyethylene glycol

Injectable

Organ culture

\begin{abstract}
A B S T R A C T
Cell delivery to the pathological intervertebral disc (IVD) has significant therapeutic potential for enhancing IVD regeneration. The development of injectable biomaterials that retain delivered cells, promote cell survival, and maintain or promote an NP cell phenotype in vivo remains a significant challenge. Previous studies have demonstrated NP cell - laminin interactions in the nucleus pulposus (NP) region of the IVD that promote cell attachment and biosynthesis. These findings suggest that incorporating laminin ligands into carriers for cell delivery may be beneficial for promoting NP cell survival and phenotype. Here, an injectable, laminin-111 functionalized poly(ethylene glycol) (PEGLM111) hydrogel was developed as a biomaterial carrier for cell delivery to the IVD. We evaluated the mechanical properties of the PEG-LM111 hydrogel, and its ability to retain delivered cells in the IVD space. Gelation occurred in approximately $20 \mathrm{~min}$ without an initiator, with dynamic shear moduli in the range of $0.9-1.4 \mathrm{kPa}$. Primary NP cell retention in cultured IVD explants was significantly higher over 14 days when cells were delivered within a PEG-LM111 carrier, as compared to cells in liquid suspension. Together, these results suggest this injectable laminin-functionalized biomaterial may be an easy to use carrier for delivering cells to the IVD.
\end{abstract}

(ㄷ) 2013 Elsevier Ltd. All rights reserved.

\section{Introduction}

Intervertebral disc (IVD) degeneration contributes to disability and symptomatic pain in affected individuals, and affects more than half the US population at some point in their lifetime [1]. A number of biological and anatomical changes are associated with IVD degeneration, initially occurring in the nucleus pulposus (NP), where degeneration is characterized by decreased cellularity, decreased water content and loss of proteoglycan deposition in the extracellular matrix (ECM) [2]. These changes in matrix composition coincide with the loss of a distinct cell population derived from the embryonic notochord $[3,4]$; therefore, it has been hypothesized

\footnotetext{
* Corresponding author. Department of Biomedical Engineering, 136 Hudson Hall, Box 90281, Durham, NC 27708, USA.

E-mail addresses: lori.setton@duke.edu, setton@duke.edu (L.A. Setton).
}

that notochordal cell disappearance may be an initiating or contributing factor to degenerative disc disease [5-7]. Since current surgical treatments of disease resulting from disc degeneration often do not restore IVD function [8] and can lead to increased mechanical load and adjacent segment disease [9], there exists significant interest in tissue engineering strategies for regenerating the IVD.

Cell transplantation to the IVD has been performed clinically [10], and aims to repopulate the disc with cells capable of synthesizing new ECM to restore function to the diseased IVD. In animal models of IVD regeneration, reimplantation of autologous disc cells or stem cells has been shown to delay degeneration, as measured by histological and radiographic changes [8,11-16]. Although the need for a biomaterial carrier to improve cell retention immediately after delivery has been demonstrated [17], few studies have evaluated the role of the carrier in long-term cell survival and retention [16]. Injectable materials for cell delivery to the IVD have received 
considerable attention since they provide a minimally invasive tissue engineering approach for enhancing IVD regeneration. Numerous hydrogels derived from natural components of the ECM, including fibrin [17-19], hyaluronan [11,20,21], and collagen $[12,22,23]$, and natural biopolymers such as chitosan [24-26] have been investigated as carriers for cell delivery to the disc. Although these naturally derived materials mimic many features of the native ECM, few peptide and protein functionalized scaffolds have been developed that can direct biological responses of cells for NP regeneration. The development of injectable biomaterials that support cell retention, cell survival and maintain or promote an NP cell phenotype in vivo remains a significant challenge.

Laminins are heterotrimeric ECM proteins consisting of $\alpha, \beta$, and $\gamma$ polypeptide chains that mediate a number of cellular functions including adhesion, survival, migration and differentiation $[27,28]$. Previous studies in our laboratory have demonstrated NP cell laminin interactions that are unique to the immature disc, suggesting that laminins may be important contributors to NP-specific cell biology. Immunohistochemistry and flow cytometry results demonstrated higher expression levels of the laminin $\alpha 5$ and $\gamma 1$ chains, laminin receptors (integrin $\alpha 3, \alpha 6, \beta 4$ subunits, CD239), and related binding proteins in NP cells as compared to cells from the adjacent anulus fibrosus [29-31]. Additional studies have shown that soft, laminin containing ECM substrates promote immature NP cell morphology, cell-cell interactions, and proteoglycan synthesis for cells of the NP [32]. Finally, immature porcine NP cells adhere to laminins in higher numbers as compared to cells from the adjacent anulus fibrosus [33]. These findings provide support for known interactions between immature NP cells and multiple laminin isoforms that regulate NP cell biology, and suggest that a soft, laminin-functionalized hydrogel may be desirable for promoting primary NP cell phenotype and biosynthesis.

Poly(ethylene glycol) (PEG) hydrogels have been widely used in tissue engineering applications since they are hydrophilic polymers that readily allow for incorporation of biological signals derived from the native ECM [34]. The non-fouling nature of PEG, combined with its tunable mechanical properties, allows for control over biological signal presentation and hydrogel stiffness. Therefore, full length ECM-derived proteins, including collagen, fibrinogen and laminin, have been covalently coupled to PEG hydrogels for a variety of tissue engineering applications and shown to influence cell behavior in three dimensions [35-38]. The majority of these studies rely on photocrosslinking of acrylate functional groups on PEGylated proteins and PEG-multiacrylates to form three-dimensional hydrogels; however, conjugate addition between free thiols and PEG-acrylate or PEG-vinylsulfone allows gelation to occur in situ under physiological conditions $[39,40]$ without the need for UV light. This chemistry enables a utility of the PEG-crosslinking hydrogel that is more suitable for the delivery of cells in vivo.

The objective of this work was to develop an injectable lamininfunctionalized PEG hydrogel with tunable mechanical properties, and to assess its potential use as a carrier for cell-based IVD regeneration. We describe here the synthesis and characterization of a PEG-laminin (PEG-LM111) conjugate with functional acrylates for crosslinking, and subsequent hydrogel formation via the addition of PEG-dithiol and PEG-octoacrylate. Additionally, we describe the generation of luciferase expressing primary NP cells, and the delivery of these cells to the disc space within our injectable biomaterial carrier.

\section{Materials \& methods}

\subsection{PEG-laminin (PEG-LM111) conjugate synthesis and characterization}

Laminin-111 (LM111, Trevigen ${ }^{\circledR}$, Gaithersburg, MD) was PEGylated with acrylatePEG-N-hydroxysuccinimide (Ac-PEG-NHS, MW $=10 \mathrm{kDa}$, Creative PEGworks,
Winston Salem, NC) to introduce functional acrylate groups for crosslinking. LM111 was dialyzed into a $0.1 \mathrm{~m}$ sodium bicarbonate buffer, $\mathrm{pH} 8.5$, and diluted to a concentration of $2 \mathrm{mg} / \mathrm{ml}$. Ac-PEG-NHS was solubilized in ice cold $0.1 \mathrm{M}$ sodium bicarbonate buffer and added to LM111 solution at 10:1, 25:1,100:1 or 500:1 M ratio of AcPEG-NHS to LM111. Reactions were carried out for $2 \mathrm{~h}$ at room temperature. Precursor PEG-LM111 conjugate solutions were dialyzed against $1 \mathrm{x}$ PBS to remove any unreacted Ac-PEG-NHS. LM111 concentration in each PEG-LM111 conjugate precursor solution was determined by measuring the absorbance at $280 \mathrm{~nm}$, and conjugates were stored at $-80{ }^{\circ} \mathrm{C}$ until further use.

A TNBS (2,4,6-trinitrobenzene sulfonic acid) assay [41] was modified to measure the free amino groups in each PEG-LM111 conjugate compared to unmodified protein, and to estimate the degree of modification due to Ac-PEG-NHS substitution. Each PEG-LM111 conjugate and unmodified LM111 were diluted to $500 \mu \mathrm{g} / \mathrm{ml}$ in PBS, which was determined to be within the linear range for LM111 using this assay. TNBS was diluted to $0.01 \%$ in $0.1 \mathrm{~m}$ sodium bicarbonate buffer, $\mathrm{pH}$ 8.5. Samples (PEG-LM111 conjugates or LM111) were mixed with $0.01 \%$ TNBS at a $1: 1$ ratio by volume, and incubated for $30 \mathrm{~min}$ at $60{ }^{\circ} \mathrm{C}$. Absorbance was measured at $340 \mathrm{~nm}$ using a microplate reader (Enspire, PerkinElmer, Waltham, MA) and used to calculate degree of modification (\%): $100 \times\left\{1-\left(\left(\mathrm{A}_{340}\right.\right.\right.$ PEG-LM111)/(A 340 LM111) $\left.)\right\}$. An ANOVA was performed to analyze degree of modification, using Tukey's post hoc test $(p<0.01, n=3)$ to detect differences between PEG-LM111 conjugates synthesized with different Ac-PEG-NHS to LM111 ratios.

\subsection{Cell isolation and culture}

Cells isolated from the porcine NP have a unique notochordal phenotype, and were therefore used to evaluate both PEG-LM111 conjugate bioactivity and for in vitro and in vivo cell delivery experiments. Lumbar spines were obtained from pigs shortly after sacrifice (L1-L5, 4-7 months, Nahunta Pork Outlet, Raleigh NC). Cells were isolated from the NP regions of IVDs by enzymatic digestion [42] and cultured in monolayer for 3-7 days in culture media (Ham's F-12 media supplemented with $10 \%$ fetal bovine serum (FBS), $10 \mathrm{~mm}$ HEPES, $100 \mathrm{U} / \mathrm{ml}$ penicillin, and $100 \mathrm{U} / \mathrm{ml}$ streptomycin) prior to experiments. For control studies, cells from a lung epithelial cell line (WI26VA4, ATCC No. CCL-95-1) were cultured in monolayer $\left(37^{\circ} \mathrm{C}, 5 \% \mathrm{CO}_{2}\right.$ $20 \% \mathrm{O}_{2}$ ) with media changes every 3-4 days (Dulbecco's Modified Eagle's Medium supplemented with $10 \%$ FBS, 10 mM HEPES) prior to experiments.

\subsection{PEG-LM111 conjugate bioactivity}

To evaluate cell attachment to PEG-LM111 conjugates, wells of 96-well plates were coated with PEG-LM111 conjugates synthesized with various ratios of Ac-PEGNHS to LM111 at 5,10 , and $25 \mu \mathrm{g} / \mathrm{ml}$ LM111 by overnight incubation at $4{ }^{\circ} \mathrm{C}$. Coated wells were blocked with $3.75 \%$ bovine serum albumin (BSA) for $3 \mathrm{~h}$ at $37^{\circ} \mathrm{C}$ to prevent non-specific adhesion. LM111 coated wells and BSA only coated wells were used as positive and negative controls, respectively. Porcine NP cells in monolayer were detached using trypsin/EDTA, washed with trypsin neutralizing solution and resuspended in serum free media. Cells ( 4000 cells/well) were allowed to adhere to the LM111 and PEG-LM111 conjugate coated surfaces for $2 \mathrm{~h}$ at $37{ }^{\circ} \mathrm{C}$. Wells were rinsed with serum free media to remove non-adherent cells, and the number of adherent cells per well was determined using CellTiterGlo ${ }^{\circledR}$ (Promega Corporation, Madison, WI) luminescent cell viability reagent. Differences in attachment numbers to PEG-LM111 conjugates at $25 \mu \mathrm{g} / \mathrm{ml}$ LM111 were detected via ANOVA with Tukey's post hoc test ( $p<0.05, n=3$ separate cell isolations).

To determine if PEG-LM111 conjugate maintains the ability to induce ERK activation upon cell adhesion, wells of 6 -well tissue culture plates were coated by overnight incubation at $4{ }^{\circ} \mathrm{C}$ with $20 \mu \mathrm{g} / \mathrm{ml}$ LM111 or PEG-LM111 that had been PEGylated at the highest ratio of Ac-PEG-NHS shown to promote cell attachment at levels similar to native protein. All wells were blocked with $3.75 \%$ BSA to prevent non-specific adhesion. Cells from a lung epithelial cell line WI26VA4 (ATCC No. CCL95-1) that had been cultured to confluence, then serum deprived for $24 \mathrm{~h}$, were seeded onto LM111 or PEG-LM111 conjugate coated surfaces $(500,000$ cells/well) WI26VA4 cells cultured in suspension served as negative controls. After 30 and $60 \mathrm{~min}$, cells were lysed with ice cold cell lysis buffer containing protease and phosphatase inhibitors (RIPA, Cell Signaling Technologies, Danvers, MA), spun down at $4{ }^{\circ} \mathrm{C}$, and cell lysates were stored at $-80^{\circ} \mathrm{C}$. Total protein concentration in each of the cell lysates was determined using the BCA Protein Assay (Thermo Scientific, Waltham, MA). All lysates were diluted in cell lysis buffer to equal concentrations of total protein and a phospho-ERK ELISA (Cell Signaling Technologies) was used to detect relative levels of phosphorylated ERK.

\subsection{PEG-LM111 hydrogel synthesis and mechanical properties}

PEG-octoacrylate (20 kDa, Creative PEGworks) and PEG-dithiol (3.4 kDa, Creative PEGworks) were dissolved separately in PEG-LM111 conjugate (25:1 Ac-PEGNHS to LM111) solutions and PBS to final concentrations of $10 \%(\mathrm{w} / \mathrm{v})$ PEG and 0 , 100 , or $500 \mu \mathrm{g} / \mathrm{mL}$ PEG-LM111 conjugate (25:1 Ac-PEG-NHS to LM111). All samples were tested in oscillatory shear under physiological conditions $\left(37^{\circ} \mathrm{C}, \mathrm{pH} 7.4\right)$ in a humidified atmosphere. Briefly, appropriate volumes of PEG-octoacrylate and PEGdithiol solutions that had been dissolved in PEG-LM111 conjugate solution were 
mixed to obtain a PEG-LM111 precursor solution containing a 1:1 ratio of acrylate to thiol groups. Solutions were mixed and immediately pipetted onto the center of a test platen ( $25 \mathrm{~mm}$ diameter) within the test chamber of a controlled stress rheometer (AR-G2, TA Instruments, New Castle, DE). The upper platen was lowered to a gap thickness of $1 \mathrm{~mm}$ and oscillatory shear was applied $\left(\omega=0.5 \mathrm{~Hz}, \gamma_{0}=0.05\right)$. The evolution of storage $\left(G^{\prime}\right)$ and loss $\left(G^{\prime \prime}\right)$ moduli were recorded over time, and used to calculate the complex shear modulus $\left(\left|G^{*}\right|\right)$. Gelation time was determined by the crossover of $G^{\prime}$ and $G^{\prime \prime}$ for each hydrogel formulation. Differences in gelation time and steady-state complex shear modulus amongst gel formulations were detected by ANOVA with Tukey's post hoc test ( $p<0.05, n=5$ per formulation).

\subsection{Generation of luciferase expressing primary NP cells}

To generate transduction media, VSV-G pseudotyped lentivirus driving constitutive expression of the luciferase2 transgene (obtained from pGL4.50; Promega, Madison, WI) under control of the EF1 $\alpha$ promoter (LVE-LUC2) was prepared in 293T cells using the standard calcium phosphate precipitation technique [43] and stored at $-80^{\circ} \mathrm{C}$ until further use. LVE-LUC2 was thawed and supernatant was centrifuged through a $100 \mathrm{kDa}$ MWCO filter (EMD Millipore, Billerica, MA). A concentrated LVELUC2 vector ( $80 \mathrm{x}$ concentration) was mixed with NP culture media (5 $\mu$ l concentrated virus per ml media) supplemented with $4 \mu \mathrm{g} / \mathrm{ml}$ polybrene ${ }^{\circledR}$ (Sigma-Aldrich, St. Louis, MO). Primary porcine NP cells were isolated as described above, plated at a density of 40,000 cells $/ \mathrm{cm}^{2}$ in culture media, and cells were allowed to adhere for $24 \mathrm{~h}$. Culture media was then aspirated from NP cells and replaced with transduction media. After $24 \mathrm{~h}$, LVE-LUC2 containing media was aspirated and fresh culture media was added. Luciferase expressing porcine NP (NP-luc) cells were cultured in monolayer with media changes every $2-3$ days.

\subsection{Cell delivery to IVD motion segments}

The effect of PEG-LM111 biomaterial on cell retention and survival in the IVD was evaluated in an organ culture model. Rat tails ( $n=8$ Sprague Dawley, 200$250 \mathrm{~g}$. Charles River, Wilmington, MA) were harvested within $3 \mathrm{~h}$ of sacrifice, skin and tendons were removed, and 7-8 IVD motion segments were obtained from each animal ( $\mathrm{C} 1-\mathrm{C} 8)$. Disc motion segments were washed three times, and placed in sterile petri dishes. A nucleotomy was performed by injecting air into the disc space via a 27 G needle. PEG-LM111 precursor solution (10\% PEG containing 1:1 acrylate to thiol ratio, $500 \mu \mathrm{g} / \mathrm{ml}$ PEG-LM111 conjugate synthesized with 25:1 Ac-PEG-NHS to LM111) was prepared as described above. NP-luc cells that had been cultured in monolayer for at least 10 days were lifted and resuspended at a concentration of $10^{6}$ cells/ml in PEG-LM111 precursor solution or PBS, and $10 \mu \mathrm{l}$ was delivered to the NP of individual caudal motion segments through a $27 \mathrm{G}$ needle. The needle was held in place for $1 \mathrm{~min}$ following cell delivery to reduce leakage. Individual motion segments were separated, placed in separate wells of a 12-well plate, and overlaid with media. Motion segments receiving no additional injection (sham) and PEGLM111 precursor solution only were used as negative controls. All motion segments were cultured out to 14 days at $37^{\circ} \mathrm{C}, 5 \% \mathrm{CO}_{2}$ and $20 \% \mathrm{O}_{2}$ in culture media ( $\mathrm{F} 12$ supplemented with $20 \% \mathrm{FBS}, 100 \mathrm{U} / \mathrm{ml}$ penicillin, and $100 \mathrm{U} / \mathrm{ml}$ streptomycin) with media changes every day.

\subsection{Cell delivery to rat tail IVD}

In vivo studies of cell persistence within the rat IVD were performed to test proof-of-concept for use of the PEG-LM111 hydrogel as a carrier for NP-luc cells. NPluc cells were delivered to the rat tail IVD either in PEG-LM111 hydrogel, PEG-only hydrogel (no PEG-LM111 conjugate) or PBS. Rats (Sprague Dawley, 200-250 g, Charles River) were anesthetized with isoflurane, and a $3 \mathrm{~cm}$ incision was made on the dorsal side of the tail to obtain access to the $\mathrm{C} 3-\mathrm{C} 4, \mathrm{C} 4-\mathrm{C} 5$ and $\mathrm{C} 5-\mathrm{C} 6$ disc space. A nucleotomy was performed at the C3-C4 and C5-C6 levels of each tail using a $25 \mathrm{G}$ needle, and the anulus fibrosus was closed with one 5-0 Vicryl ${ }^{\circledR}$ suture (Ethicon Inc., Somerville, NJ). Cells in PBS, cells in PEG-LM111 (PEG-octoacrylate and PEG-dithiol, $500 \mu \mathrm{g} / \mathrm{ml}$ PEG-LM111 conjugate), and cells in PEG-only (PEG-octoacrylate and PEG-dithiol, no PEG-LM111 conjugate) were prepared as described above. Using a Hamilton syringe with a 27G needle, $10 \mu$ of cells in PEG-LM111, cells in PBS, or cells in PEG-only was injected into the middle of the C3-C4 denucleated disc space ( $n=2$ per group). The C5-C6 disc space of the same tail received either PEG-LM111 biomaterial only or nucleotomy only (sham). Skin was closed in an interrupted manner using 4.0 nylon sutures. All procedures were approved by the IACUC and were performed in accordance with Duke University guidelines.

\subsection{Bioluminescent imaging}

Bioluminescent imaging of cell culture plates, IVD motion segments or rats that had received NP-luc cells was performed using an $\mathrm{IVIS}^{\odot}$ Kinetic, and signal intensities were quantified with Living Imaging software (PerkinElmer, Waltham, MA). For in vitro evaluation of the stability of luciferase expression by NP-luc cells in monolayer, NP-luc and control naïve NP cells were lifted at multiple time points post transduction (day 4-35), counted and suspended in culture media. These cells were added to separate wells of a 96 well black bottom tissue culture plate
(5000 cells/well). Wells were supplemented with $150 \mu \mathrm{g} / \mathrm{ml}$ D-luciferin (PerkinElmer) and imaged 10 min after addition of D-luciferin ( $5 \mathrm{~s}$ exposure time). Cell retention in rat motion segments was evaluated at multiple time points after injection of NP-luc cells using bioluminescence imaging. To evaluate cell retention in IVD motion segments, wells containing rat IVD motion segments were supplemented with $300 \mu \mathrm{g} / \mathrm{ml}$ D-luciferin and light emission was measured 2 min later (30 s exposure time). For in vivo imaging, D-luciferin $(150 \mathrm{mg} / \mathrm{kg})$ was injected intraperitoneally $15 \mathrm{~min}$ before imaging, after which rats were anesthetized with $1-$ $3 \%$ isoflurane and imaged ( 5 min exposure time). For NP-luc cells cultured in monolayer, total photons per second per cell was calculated from the total photons in a defined region of interest (ROI) drawn over each well. An ANOVA was performed to analyze light emission ( hotons/sec/cell), using Tukey's post hoc test $(p<0.05$, $n=3$ ) to detect differences between time points. For cultured IVD motion segments, an oval ROI was drawn around the entire motion segment based on a photographic image. Total photons within each ROI was obtained from the overlay luminescent image. Differences amongst cell delivery vehicles and time post injection were analyzed via two-factor ANOVA with Tukey's post hoc test $(p<0.05, n=6)$.

\section{Results}

\subsection{PEG-LM111 conjugate synthesis}

PEG-LM111 conjugates with functional acrylate groups for crosslinking were synthesized via amine reactive NHS groups (Fig. 1A). Degree of LM111 modification, as measured by the reduction in number of reactive amines in each PEG-LM111 conjugate, significantly increased with increasing ratios of Ac-PEG-NHS to LM111 in the reaction solution (Fig. 1B). A minimum 25-fold molar excess of Ac-PEG-NHS over LM111 in the PEGylation reaction was

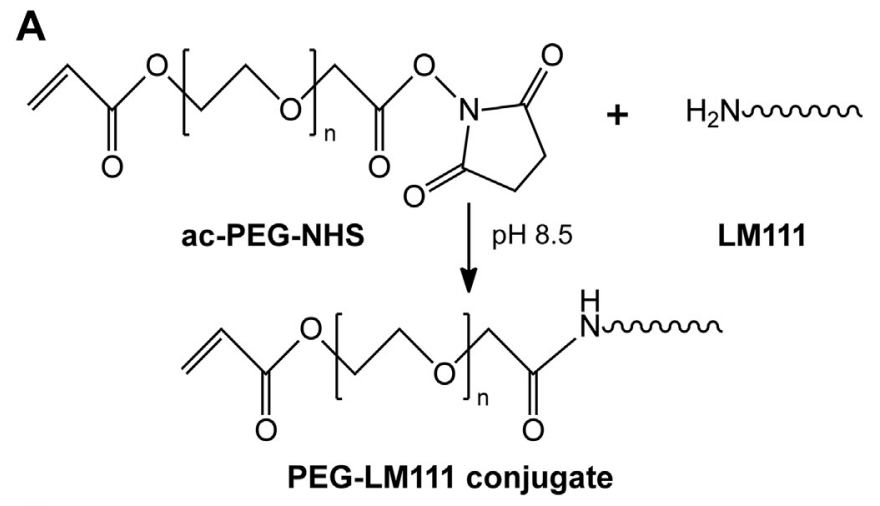

B

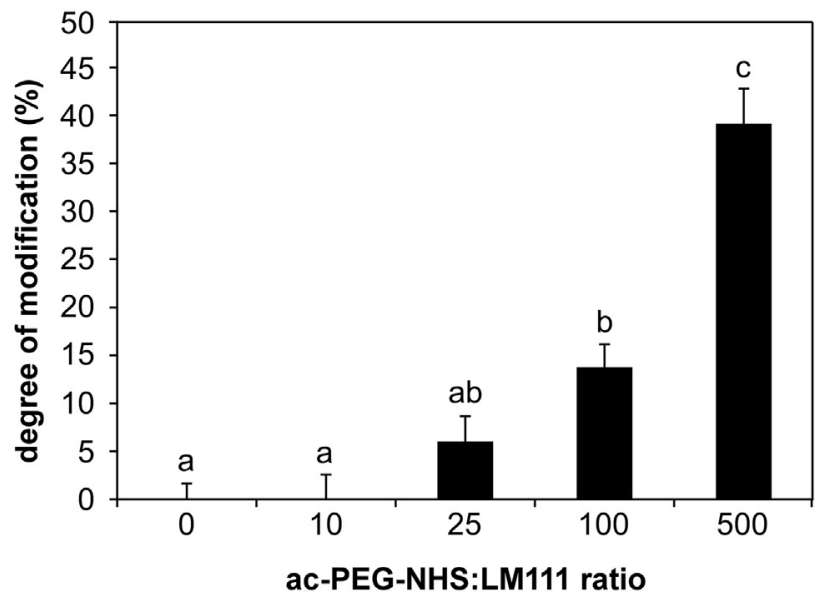

Fig. 1. PEG-LM111 conjugate synthesis and degree of modification (\%). (A) Conjugation of PEG molecules to laminin via amine reactive NHS groups. (B) Degree of modification of LM111 calculated from LM111 and PEG-LM111 conjugate absorbance at $340 \mathrm{~nm}$ using TNBS assay. Degree of modification increased with increasing molar excess of AcPEG-NHS in the PEGylation reaction (mean \pm SEM, $n=3$, conditions labeled with different letters significantly different, $p<0.01$ ). 
necessary to detect protein modification (6\%) using the TNBS assay. A 500-fold molar excess of Ac-PEG-NHS resulted in 39\% LM111 modification, which was significantly higher (ANOVA, $p<0.001$ ) than that of PEG-LM111 conjugates synthesized at 10 -fold, 25 -fold or 100 -fold molar excess Ac-PEG-NHS over LM111.

\subsection{PEG-LM111 conjugate bioactivity}

To determine the relationship between the degree of LM111 modification and level of cell attachment, porcine NP cell attachment to PEG-LM111 conjugates with varying degrees of modification was assessed. Results for NP cell adhesion to PEG-LM111 conjugates demonstrated decreased cell attachment with increasing molar excess of Ac-PEG-NHS over LM111 used in conjugate synthesis, as compared to NP cell attachment to unmodified LM111 (Fig. 2A). Cell attachment was significantly reduced (ANOVA, $p<0.001$ ) for conjugates synthesized with large molar excesses of PEG (100:1 or 500:1); however, cell attachment numbers for PEGLM111 conjugate synthesized with a 25:1 ratio of Ac-PEG-NHS to LM111 were greater than $89 \%$ of values for NP cell attachment to native LM111. Therefore, PEG-LM111 conjugate synthesized at a 25fold molar excess of Ac-PEG-NHS over LM111, which corresponds to $6 \%$ degree of modification by TNBS, was used for all subsequent experiments.

To further verify bioactivity of PEG-LM111 conjugate with a low degree of modification, we investigated activation of the MAPK/ ERK signaling pathway subsequent to cell attachment to LM111 and PEG-LM111 conjugate. Since it has been shown that cell attachment to LM111 leads to ERK activation in a lung epithelial cell line (WI26VA4) [44], we hypothesized that a PEG-LM111 conjugate capable of maintaining levels of cell adhesion similar to that of native LM111 would also support ERK phosphorylation. As shown in Fig. 2B, LM111 induced a 3-fold increase in phosphorylated ERK over cells cultured in suspension after $30 \mathrm{~min}$ and a 1.6-fold increase after 60 min. PEG-LM111 conjugate induced similar levels of phosphorylated ERK as compared to LM111 with 2.8-fold and 1.7-fold higher levels of ERK phosphorylation over negative controls after 30 and $60 \mathrm{~min}$, respectively.

\subsection{PEG-LM111 hydrogel mechanical properties}

When PEG-LM111 conjugate solution or PBS was mixed with PEG-octoacrylate and PEG-dithiol under physiological conditions ( $37^{\circ} \mathrm{C}, \mathrm{pH} 7.4$ ), hydrogels formed in less than $25 \mathrm{~min}$ by the reaction of thiols and acrylates, as measured by the point of crossover of $G^{\prime}$ and $G^{\prime \prime}$ (Fig. 3A). For hydrogels containing $500 \mu \mathrm{g} / \mathrm{ml}$ PEG-LM111 conjugate, the gel point occurred in less than $14 \mathrm{~min}$. PEG-only (no PEG-LM111 conjugate) and PEG-LM111 hydrogel mechanical properties continued to evolve for approximately $1 \mathrm{~h}$. The final gel stiffness $\left(\left|G^{*}\right|\right)$ increased with increasing concentrations of PEGLM111 conjugate in the precursor solution (Fig. 3B).

\subsection{Cell delivery to IVD motion segments}

To track cells following delivery to the IVD using in vivo bioluminescent imaging, it was necessary to verify that NP-luc cells maintain luciferase expression for at least 4 weeks post transduction. Luciferase expression in NP-luc cells cultured in monolayer increased approximately 3.5 -fold from day 4 to day 35 post transduction; however, there was no significant difference in expression between day 24 and day 35 (ANOVA, $p>0.05$ ) (Fig. 4).

Bioluminescence imaging was utilized to examine the effect of PEG-LM111 hydrogel carrier on NP-luc cell retention and survival in IVD motion segments. The total number of photons in a defined region of interest (ROI) was quantified at multiple time points following cell injection (Fig. 5B). Cell retention in IVD explants was significantly higher (ANOVA, $p<0.001$ ) over 14 days in culture when cells were delivered within a PEG-LM111 hydrogel carrier, as compared to cells in PBS (Fig. 5A). At 30 min post injection, bioluminescent signal was 13 -fold higher when cells were delivered to IVDs in PEG-LM111 biomaterial; however, signal rapidly decayed over the first $24 \mathrm{~h}$ of culture for both groups. NP-luc cells in IVD
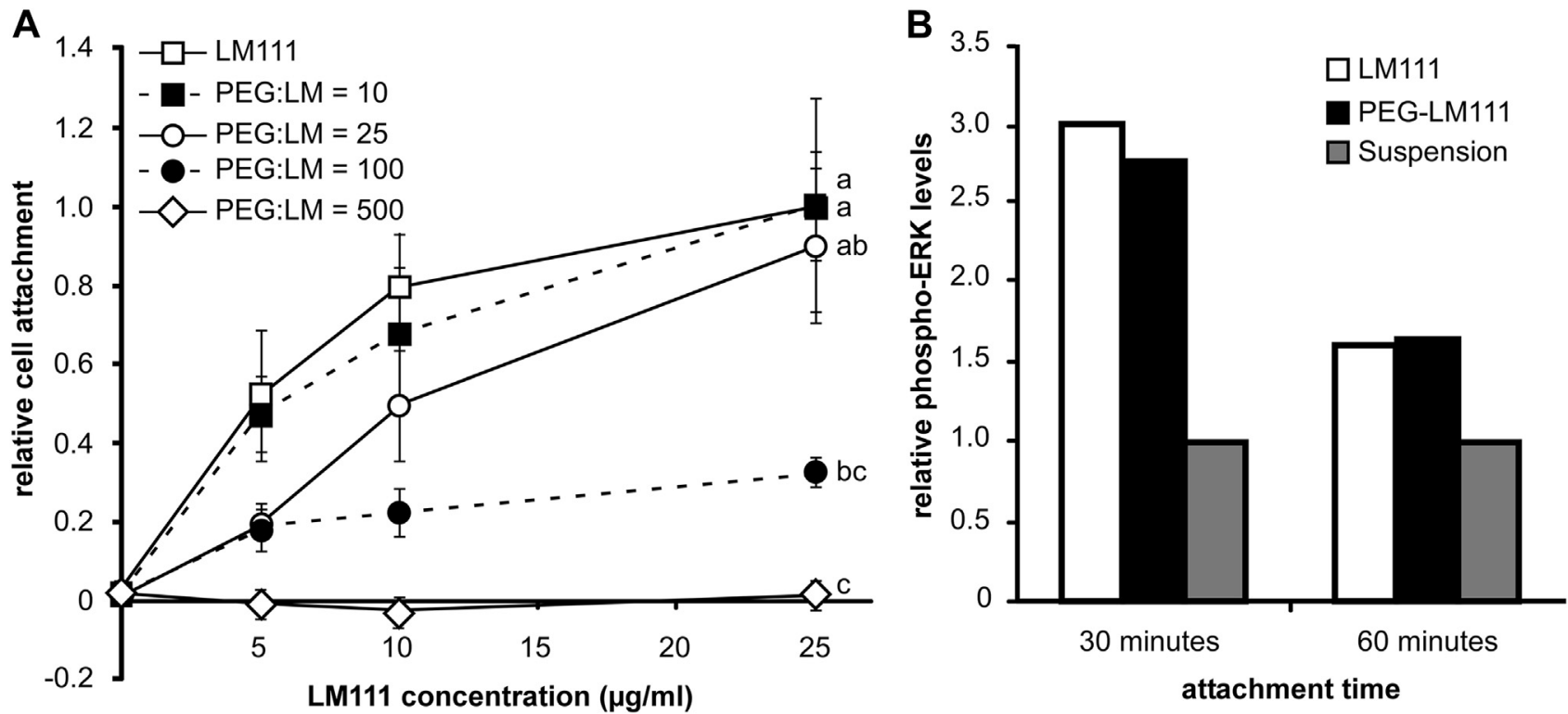

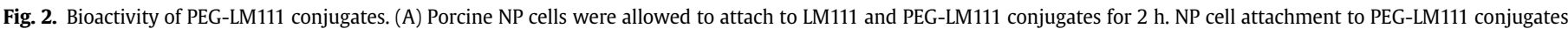

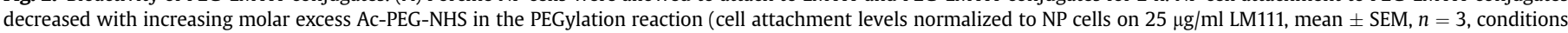

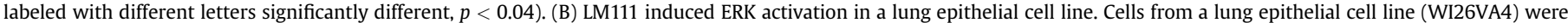

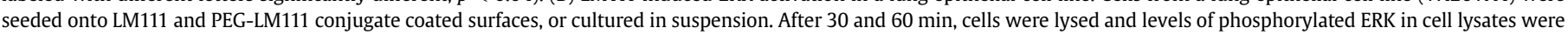
compared using ELISA. 

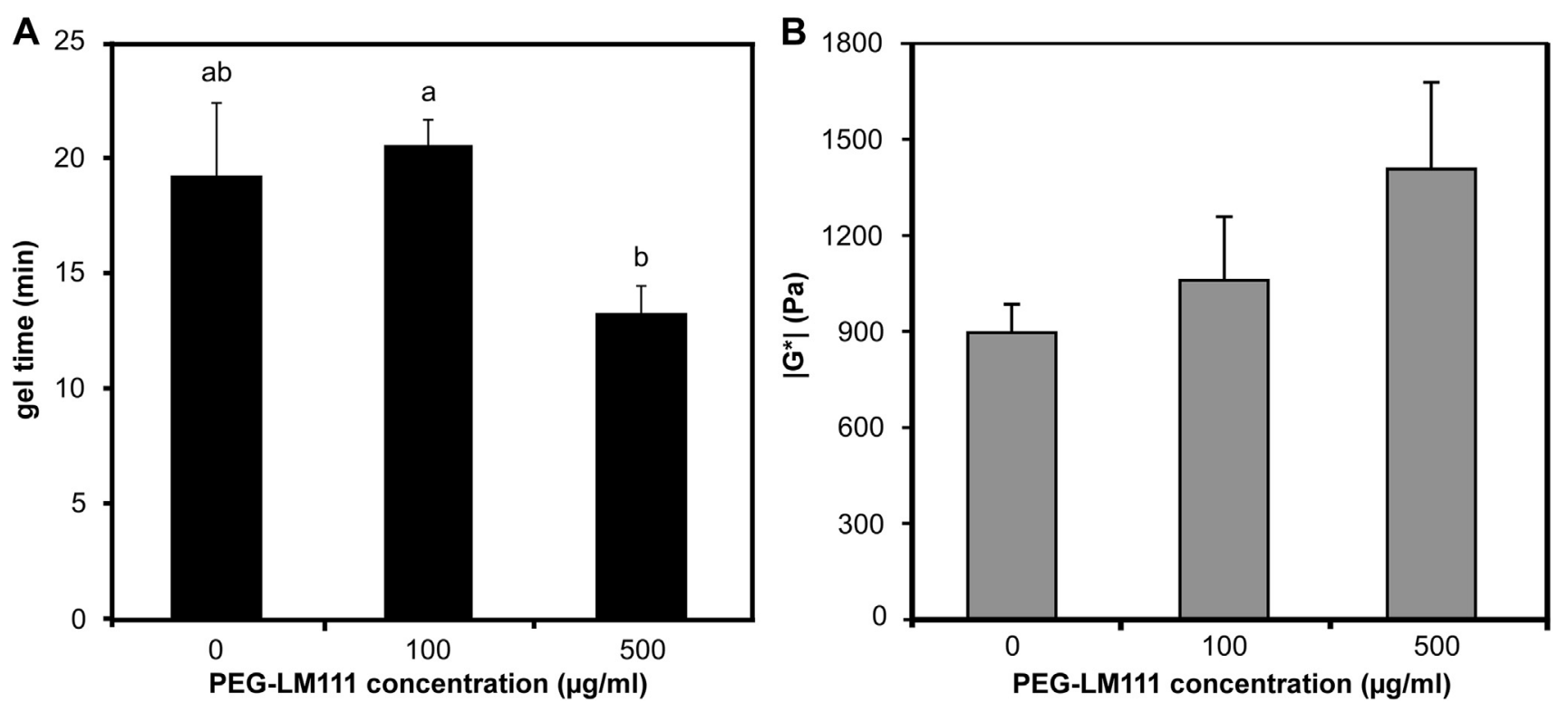

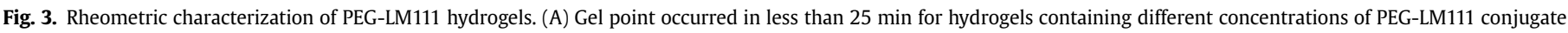

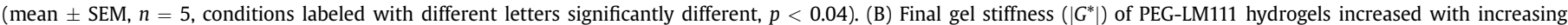
concentration of PEG-LM111 conjugate in the precursor solution.

explants could be detected via bioluminescent imaging for at least 14 days post injection.

\subsection{In vivo cell delivery to rat IVD}

Preliminary studies were performed to determine if NP-luc cells, delivered to the rat tail IVD in either PBS, PEG-LM111 hydrogel, or PEG-only hydrogel, could be detected via in vivo imaging. Cells could be detected in the disc space 15 min after intraperitoneal injection of luciferin (Fig. 6). For all animals, maximum luminescent signal did not exceed 1000 counts over a 5 min exposure time, possibly due to a low number of delivered cells, low luciferase expression levels, poor luciferin distribution to the tail, and signal attenuation by the collagenous tail skin.

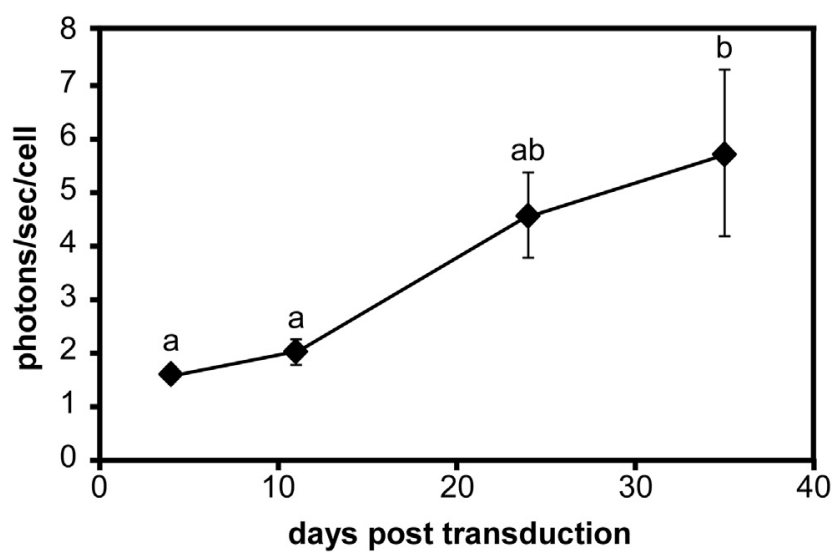

Fig. 4. Luciferase activity in cultured porcine NP cells. NP cells transduced with lentivirus encoding for firefly luciferase were cultured for 35 days post transduction. At each time point, photons per second per cell was calculated from measured bioluminescent intensity $10 \mathrm{~min}$ after addition of luciferin to the media (mean $\pm \operatorname{SEM}, n=3$, conditions labeled with different letters significantly different, $p<0.03$ ).

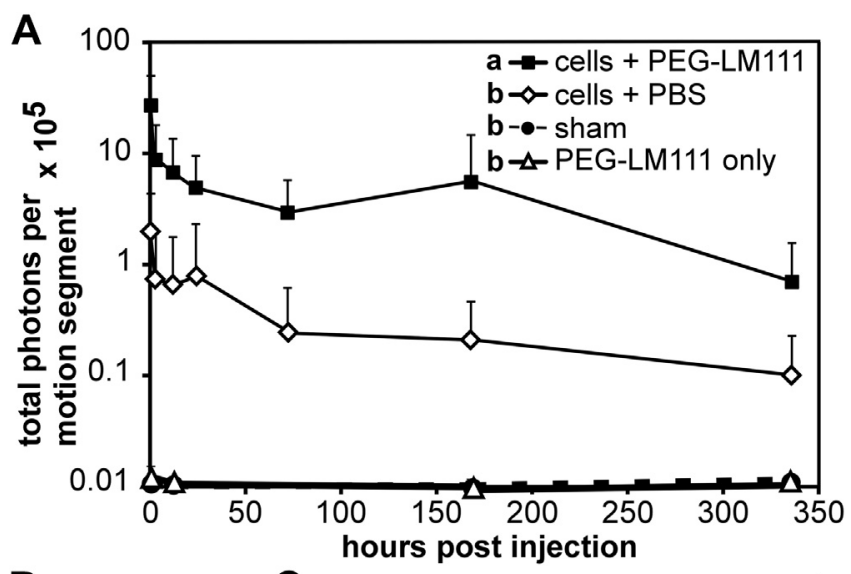

B

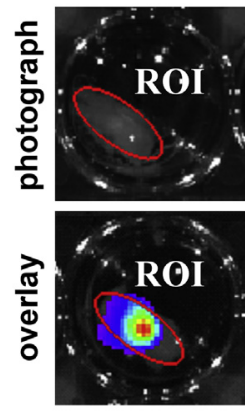

C

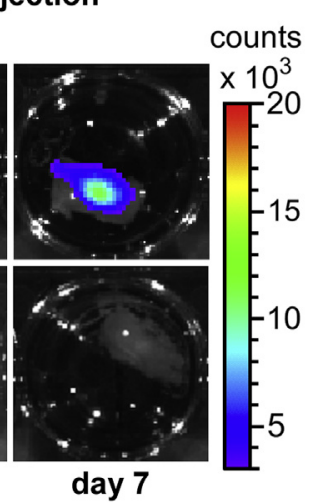

Fig. 5. Cell retention in IVD motion segments. (A) NP-luc cells were delivered to rat IVD motion segments and explants were cultured for 14 days. Cell retention, as measured by total photons per motion segment, was higher for cells delivered in PEGLM111 biomaterial as compared to cells delivered in PBS (mean \pm SEM, $n=6$, conditions labeled with different letters significantly different, $p<0.001$ ). (B) Region of interest (ROI) defined on photographic image of motion segment (top) used quantify total photons per motion segment in overlay image (bottom). (C) Representative images of IVD motion segments 30 min and 7 days post cell injection within PEG-LM111 biomaterial carrier (top) or PBS (bottom). 


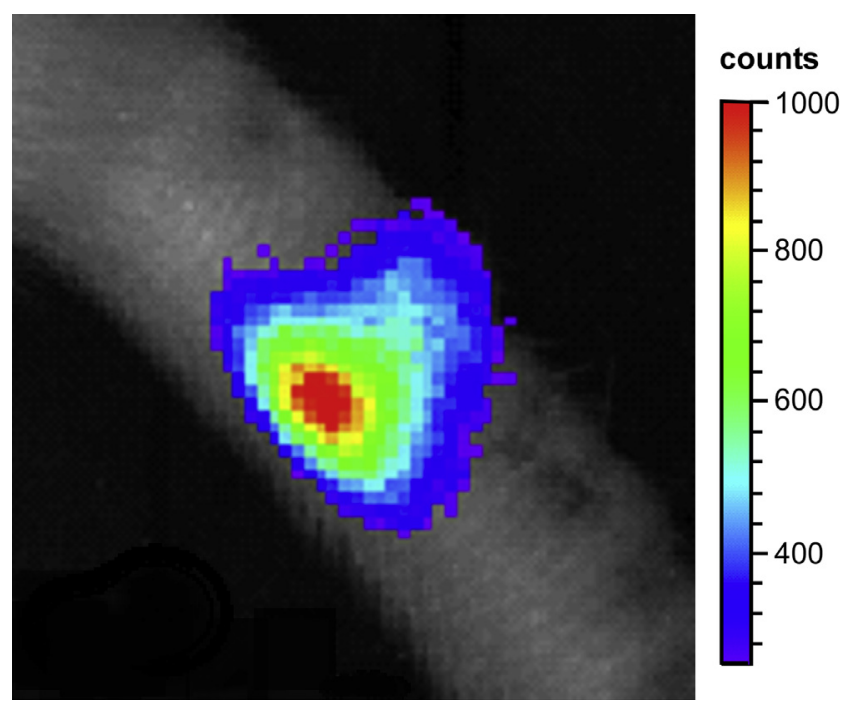

Fig. 6. In vivo bioluminescent imaging $15 \mathrm{~h}$ after $1 \times 10^{6} \mathrm{NP}$-luc cells were injected into the rat tail disc space. Luciferin $(150 \mathrm{mg} / \mathrm{kg}$ ) was injected intraperitoneally $15 \mathrm{~min}$ before imaging. Rat was anesthetized with $2 \%$ isoflurane during imaging $(5 \mathrm{~min}$ exposure time).

\section{Discussion}

The goal of this work was to develop an injectable, lamininfunctionalized hydrogel as a biomaterial carrier for cell delivery to the IVD. Laminin presenting substrates have previously been shown to promote the morphology and phenotype of immature NP cells in vitro, and to promote increased cell attachment and elevated glycosaminoglycan synthesis for primary NP cells in culture [32,33]. For these reasons, a three-dimensional biomaterial carrier presenting laminin was developed as a carrier for primary NP cells. Conjugation of the laminin protein, LM111 to synthetic PEGs was performed with varying levels of protein modification in order to identify a formulation that could maintain LM111 functionality, and appropriate cell-laminin interactions. Results showed the degree of laminin modification increased with increasing molar excess of Ac-PEG-NHS in the conjugate synthesis reaction [45], up to a maximum value of $40 \%$ primary amine modification with a 500-fold molar excess of Ac-PEG-NHS. Primary NP cell adhesion to PEG-LM111 conjugates significantly decreased with increasing degree of PEGylation, with no cells adhering to PEG-LM111 conjugate with the highest degree of modification. This finding was expected as PEGylation of therapeutic compounds has been shown to modify their structure and bioactivity [45]. Nevertheless, low levels of PEGylation (up to 25:1 Ac-PEG-NHS to LM111) did not significantly reduce NP cell adhesion to PEG-LM111 conjugates, nor inhibit MAPK/ERK activation upon binding to the modified protein. This low level of laminin modification still enabled the PEG-LM111 conjugate to participate in a gelation crosslinking reaction that was shown to be effective in promoting cell entrapment. Together, these findings demonstrate that LM111 PEGylation via a heterobifunctional Ac-PEG-NHS allows for the additional of functional acrylates groups onto native LM111 without significantly altering the protein's ability to mediate cellular function.

PEG-LM111 conjugates were crosslinked to form hydrogels upon the addition of PEG-octoacrylate and PEG-dithiol via a Michael type addition reaction without the need for an initiator, as has been shown for PEG hydrogels designed for protein delivery [39]. Gelation occurred within 20 min under physiological conditions independent of the concentration of PEG-LM111 conjugate in the precursor solution. The reaction was thus considered to provide for an acceptable working time for cell delivery in vivo since it allows time for cell suspension and accurate needle placement within the disc space [46]. The dynamic stiffness of PEG-LM111 hydrogels in shear increased slightly from $\sim 0.9 \mathrm{kPa}$ to $\sim 1.5 \mathrm{kPa}$ with increasing concentration of PEG-LM111 conjugate in the precursor solutions, all values that are only slightly lower than values reported previously for the dynamic shear moduli of human NP (7.4-19.8 kPa) [47,48]. Stiffness can be readily manipulated to achieve a targeted goal by modifying the amount of PEG within the crosslinked hydrogel. In a prior report of PEG-laminin hydrogels crosslinked by photopolymerization, hydrogel stiffness decreased with increasing concentration of the PEG-laminin conjugate precursor solutions [38], a finding that differs from that observed in the current study. This difference likely reflects intrinsic differences between stepgrowth and chain-growth polymerization reactions in the two different systems; our finding suggests that high concentrations of PEG-LM111 conjugate in the precursor solution reduces the number of non-idealities in the crosslinked hydrogel network, which are introduced in hydrogels formed by Michael-type addition reactions at low PEG concentrations [49]. This is a feature that can be modulated to achieve a targeted outcome through biomaterial design if it should become desirable to modulate the porosity or stiffness, for example, to achieve a specific set of biological outcomes [50]. Here our goal was to obtain a material that retained laminin functionality, provided good working characteristics, and physical properties that approximated that of the native NP. Overall, these findings suggest this laminin-functionalized biomaterial can provide an injectable carrier for delivering cells to the IVD.

Many biomaterials have been investigated as carriers for cell delivery to the IVD in numerous animal models of disc degeneration [17]. While solid scaffolds are widely used to support IVD cell growth ex vivo [51-53], we have focused in this study on injectable, in situ polymerizing three-dimensional hydrogels that could maintain the rounded phenotype for NP cells. Naturally derived biopolymers and self-polymerizing biomaterials such as collagen, chitosan and fibrin are most commonly utilized for NP regeneration, although the physical properties and chemical composition of these biomaterials is not easily manipulated [54]. Chemical crosslinking of alginate is the most commonly used approach to promote a rounded phenotype for NP cells and is the standard for cell culture studies [55]. More recently, photocrosslinking of alginate has been proposed as a means to manipulate a three-dimensional matrix for NP cells [56], and a self-assembling peptide hydrogel has been similarly investigated [57]. Few injectable biomaterials have presented functional ECM ligands that can retain native NP cell-matrix interactions and facilitate cell-mediated matrix remodeling, which may be important for promoting long-term cell survival and biosynthesis.

While prior studies have established the importance of a biomaterial carrier for improved cell transfer to the disc space immediately following delivery [17], little is known of the role of the biomaterial carrier in promoting long-term cell retention and survival [16]. Studies were performed here to test for an ability of the newly synthesized PEG-LM111 biomaterial to promote NP cell retention and survival in the disc space, by following luciferase expression for immature primary NP cells delivered via needle injection to the disc space in an organ culture model. The optimized PEG-LM111 hydrogel significantly improved primary NP cell retention and survival as compared to cells delivered in an uncrosslinked, liquid suspension. Thirty minutes after injection, bioluminescent signal was 13-fold higher when cells were delivered in a PEG-LM111 biomaterial, suggesting that cell delivery in the absence of a solid-setting carrier may result in dramatic cell loss [17]. These findings suggest that an optimized PEG-LM111 hydrogel is able to crosslink in situ and significantly improve upon the 
retention of delivered cells. The PEG-LM111 hydrogel was unable to inhibit complete cell loss, however, as cells continued to die or migrate from the disc space over the first $24 \mathrm{~h}$ after delivery, likely due to a high intra-discal pressure that induced extrusion through the injection site. This highlights the need for annular repair techniques in addition to biomaterials for cell delivery, or highstrength crosslinked biomaterial carriers, that may need to be employed to slow or eliminate cell loss following delivery [58].

Cell delivery to the disc space has been performed in animal models of disc degeneration and in human subjects for more than a decade [8]. Cell retention within the disc space is difficult to follow in the living subject, particularly for clinical studies that rely upon minimal modification of delivered cells [31]. Much of the work studying cell delivery to the disc space in animal models has relied on histological techniques subsequent to animal sacrifice at specific time points, which precludes in vivo assessment and longitudinal tracking of cell therapy. In a recent study, investigators demonstrated the feasibility of bioluminescence imaging for monitoring the viability of luciferase expressing cells within the lumbar spine in a rodent model up to 14 days after cell implantation [59]. Here, we performed preliminary studies to determine if similar in vivo bioluminescent imaging could be used to track NP-luc cells delivered to the rat caudal IVD. Although cells could be detected in the disc space for up to 7 days following delivery, bioluminescent signal intensity was extremely low even over long exposure times. Photon emission at the tissue surface depends on the number of delivered cells, the photon flux per cell (luciferin expression levels and luciferin availability), and the signal attenuation by the tissue [60]. Additionally, intraperitoneal injection of luciferin may not be suitable for evaluating luciferase expressing cells in the caudal discs and alternate luciferin delivery routes may need to be explored. Therefore, the very low bioluminescent signals that we reported from in vivo imaging are likely due to a combination of factors, including our delivery of comparatively low cell numbers to the disc space, and other limitations related to bioluminescence imaging.

\section{Conclusions}

We report here the development and characterization of an injectable laminin-functionalized PEG hydrogel as a biomaterial carrier for cell delivery to the IVD. The findings from this study demonstrate the ability of PEG-LM111 hydrogels to crosslink under physiological conditions without the need for an initiator. Additionally, delivery within our PEG-LM111 hydrogel significantly improved primary NP cell retention in the disc space as compared to cells delivered without a carrier in an organ culture model. Bioluminescence imaging for evaluating the effect of our carrier on persistence and viability of delivered cells in vivo merits further investigation. In summary, these findings suggest that this lamininfunctionalized hydrogel may be a useful carrier for cell-based strategies aimed at regenerating the IVD.

\section{Acknowledgments}

This work was completed with support from NIH Grants R01AR047442, R01EB002263, T32GM008555, F32AR063012, R01AR048852, and a Duke-NUS Medical Student Scholarship. The authors thank Devin T. Bridgen, Gregory M. Palmer and Stephen Johnson for their assistance with the surgical procedures.

\section{References}

[1] Owens PL, Woeltje M, Mutter R. Emergency department visits and inpatient stays related to back problems, 2008: statistical brief \#105. Healthcare Cost and Utilization Project (HCUP) statistical briefs. Rockville (MD) 2006.
[2] Lyons G, Eisenstein SM, Sweet MBE. Biochemical-changes in intervertebraldisk degeneration. Biochim Biophys Acta 1981;673:443-53.

[3] Walmsley R. The development and growth of the intervertebral disc. Edinb Med J 1953;60:341-64.

[4] Wolfe HJ, Putschar WG, Vickery AL. Role of the notochord in human intervetebral disk. I. Fetus and infant. Clin Orthop Relat Res 1965;39:205-12.

[5] Oegema Jr TR. The role of disc cell heterogeneity in determining disc biochemistry: a speculation. Biochem Soc Trans 2002;30:839-44.

[6] Hunter CJ, Matyas JR, Duncan NA. The notochordal cell in the nucleus pulposus: a review in the context of tissue engineering. Tissue Eng 2003;9: 667-77.

[7] Risbud MV, Schaer TP, Shapiro IM. Toward an understanding of the role of notochordal cells in the adult intervertebral disc: from discord to accord. Dev Dyn 2010;239:2141-8.

[8] Sakai D. Future perspectives of cell-based therapy for intervertebral disc disease. Eur Spine J 2008;17(Suppl. 4):452-8.

[9] Phillips FM, Reuben J, Wetzel FT. Intervertebral disc degeneration adjacent to a lumbar fusion. An experimental rabbit model. J Bone Jt Surg Br 2002;84: 289-94.

[10] Meisel HJ, Siodla V, Ganey T, Minkus Y, Hutton WC, Alasevic OJ. Clinical experience in cell-based therapeutics: disc chondrocyte transplantation a treatment for degenerated or damaged intervertebral disc. Biomol Eng 2007;24:5-21.

[11] Crevensten G, Walsh AJ, Ananthakrishnan D, Page P, Wahba GM, Lotz JC, et al. Intervertebral disc cell therapy for regeneration: mesenchymal stem cell implantation in rat intervertebral discs. Ann Biomed Eng 2004;32:430-4.

[12] Sakai D, Mochida J, Yamamoto Y, Nomura T, Okuma M, Nishimura K, et al. Transplantation of mesenchymal stem cells embedded in atelocollagen gel to the intervertebral disc: a potential therapeutic model for disc degeneration. Biomaterials 2003;24:3531-41.

[13] Gruber HE, Johnson TL, Leslie K, Ingram JA, Martin D, Hoelscher G, et al. Autologous intervertebral disc cell implantation: a model using Psammomys obesus, the sand rat. Spine 2002;27:1626-33.

[14] Ganey T, Libera J, Moos V, Alasevic O, Fritsch KG, Meisel HJ, et al. Disc chondrocyte transplantation in a canine model: a treatment for degenerated or damaged intervertebral disc. Spine 2003;28:2609-20.

[15] Sakai D, Mochida J, Iwashina T, Watanabe T, Nakai T, Ando K, et al. Differentiation of mesenchymal stem cells transplanted to a rabbit degenerative disc model: potential and limitations for stem cell therapy in disc regeneration. Spine 2005;30:2379-87.

[16] Leckie SK, Sowa GA, Bechara BP, Hartman RA, Coelho JP, Witt WT, et al. Injection of human umbilical tissue-derived cells into the nucleus pulposus alters the course of intervertebral disc degeneration in vivo. Spine J 2013;13: 263-72.

[17] Bertram H, Kroeber M, Wang H, Unglaub F, Guehring T, Carstens C, et al. Matrix-assisted cell transfer for intervertebral disc cell therapy. Biochem Biophys Res Commun 2005;331:1185-92.

[18] Omlor GW, Bertram H, Kleinschmidt K, Fischer J, Brohm K, Guehring T, et al. Methods to monitor distribution and metabolic activity of mesenchymal stem cells following in vivo injection into nucleotomized porcine intervertebral discs. Eur Spine J 2010;19:601-12.

[19] Saldanha KJ, Piper SL, Ainslie KM, Kim HT, Majumdar S. Magnetic resonance imaging of iron oxide labelled stem cells: applications to tissue engineering based regeneration of the intervertebral disc. Eur Cell Mater 2008;16:17-25.

[20] Peroglio M, Grad S, Mortisen D, Sprecher CM, Illien-Junger S, Alini M, et al. Injectable thermoreversible hyaluronan-based hydrogels for nucleus pulposus cell encapsulation. Eur Spine J 2012;21(Suppl. 6):S839-49.

[21] Revell PA, Damien E, Di Silvio L, Gurav N, Longinotti C, Ambrosio L. Tissue engineered intervertebral disc repair in the pig using injectable polymers. J Mater Sci Mater Med 2007;18:303-8.

[22] Sakai D, Mochida J, Iwashina T, Hiyama A, Omi H, Imai M, et al. Regenerative effects of transplanting mesenchymal stem cells embedded in atelocollagen to the degenerated intervertebral disc. Biomaterials 2006;27:335-45.

[23] Collin EC, Grad S, Zeugolis DI, Vinatier CS, Clouet JR, Guicheux JJ, et al. An injectable vehicle for nucleus pulposus cell-based therapy. Biomaterials 2011;32:2862-70.

[24] Cheng YH, Yang SH, Su WY, Chen YC, Yang KC, Cheng WT, et al. Thermosensitive chitosan-gelatin-glycerol phosphate hydrogels as a cell carrier for nucleus pulposus regeneration: an in vitro study. Tissue Eng Part A 2010;16: 695-703.

[25] Dang JM, Sun DD, Shin-Ya Y, Sieber AN, Kostuik JP, Leong KW. Temperatureresponsive hydroxybutyl chitosan for the culture of mesenchymal stem cells and intervertebral disk cells. Biomaterials 2006;27:406-18.

[26] Roughley P, Hoemann C, DesRosiers E, Mwale F, Antoniou J, Alini M. The potential of chitosan-based gels containing intervertebral disc cells for nucleus pulposus supplementation. Biomaterials 2006;27:388-96.

[27] Miner JH, Yurchenco PD. Laminin functions in tissue morphogenesis. Annu Rev Cell Dev Biol 2004;20:255-84.

[28] Ekblom P, Lonai P, Talts JF. Expression and biological role of laminin-1. Matrix Biol 2003;22:35-47.

[29] Gilchrist CL, Chen J, Richardson WJ, Loeser RF, Setton LA. Functional integrin subunits regulating cell-matrix interactions in the intervertebral disc. J Orthop Res 2007;25:829-40.

[30] Chen J, Jing L, Gilchrist CL, Richardson WJ, Fitch RD, Setton LA. Expression of laminin isoforms, receptors, and binding proteins unique to nucleus pulposus cells of immature intervertebral disc. Connect Tissue Res 2009;50:294-306. 
[31] Nettles DL, Richardson WJ, Setton LA. Integrin expression in cells of the intervertebral disc. J Anat 2004;204:515-20.

[32] Gilchrist CL, Darling EM, Chen J, Setton LA. Extracellular matrix ligand and stiffness modulate immature nucleus pulposus cell-cell interactions. PloS One 2011;6:e27170.

[33] Gilchrist CL, Francisco AT, Plopper GE, Chen J, Setton LA. Nucleus pulposus cell-matrix interactions with laminins. Eur Cell Mater 2011;21:523-32.

[34] Drury JL, Mooney DJ. Hydrogels for tissue engineering: scaffold design variables and applications. Biomaterials 2003;24:4337-51.

[35] Zhu J. Bioactive modification of poly(ethylene glycol) hydrogels for tissue engineering. Biomaterials 2010;31:4639-56.

[36] Dikovsky D, Bianco-Peled H, Seliktar D. Defining the role of matrix compliance and proteolysis in three-dimensional cell spreading and remodeling. Biophys J 2008;94:2914-25.

[37] Peyton SR, Kim PD, Ghajar CM, Seliktar D, Putnam AJ. The effects of matrix stiffness and RhoA on the phenotypic plasticity of smooth muscle cells in a 3-D biosynthetic hydrogel system. Biomaterials 2008;29:2597-607.

[38] Marquardt L, Willits RK. Neurite growth in PEG gels: effect of mechanical stiffness and laminin concentration. J Biomed Mater Res A 2011;98:1-6.

[39] Elbert DL, Pratt AB, Lutolf MP, Halstenberg S, Hubbell JA. Protein delivery from materials formed by self-selective conjugate addition reactions. J Control Release 2001;76:11-25.

[40] Pratt AB, Weber FE, Schmoekel HG, Muller R, Hubbell JA. Synthetic extracellular matrices for in situ tissue engineering. Biotechnol Bioeng 2004;86:27-36.

[41] Habeeb AF. Chemical evaluation of conformational differences in native and chemically modified proteins. Biochim Biophys Acta 1966;115:440-54.

[42] Chen J, Baer AE, Paik PY, Yan W, Setton LA. Matrix protein gene expression in intervertebral disc cells subjected to altered osmolarity. Biochem Biophys Res Commun 2002;293:932-8.

[43] Salmon P, Trono D. Production and titration of lentiviral vectors, Curr Protoc Neurosci. 2006; Chapter 4:Unit 4.21.

[44] Ferletta M, Kikkawa Y, Yu H, Talts JF, Durbeej M, Sonnenberg A, et al. Opposing roles of integrin alpha6Abeta1 and dystroglycan in laminin-mediated extracellular signal-regulated kinase activation. Mol Biol Cell 2003; 14:2088-103.

[45] Veronese FM, Mero A. The impact of PEGylation on biological therapies. BioDrugs: Clin Immuno Biopharm Gene Ther 2008;22:315-29.

[46] Boyd LM, Carter AJ. Injectable biomaterials and vertebral endplate treatment for repair and regeneration of the intervertebral disc. Eur Spine J 2006;15(Suppl. 3): S414-21.
[47] Iatridis JC, Setton LA, Weidenbaum M, Mow VC. The viscoelastic behavior of the non-degenerate human lumbar nucleus pulposus in shear. J Biomech 1997;30:1005-13.

[48] Iatridis JC, Weidenbaum M, Setton LA, Mow VC. Is the nucleus pulposus a solid or a fluid? Mechanical behaviors of the nucleus pulposus of the human intervertebral disc. Spine 1996;21:1174-84.

[49] Metters A, Hubbell J. Network formation and degradation behavior of hydrogels formed by Michael-type addition reactions. Biomacromolecules 2005;6:290-301.

[50] Nettles DL, Haider MA, Chilkoti A, Setton LA. Neural network analysis identifies scaffold properties necessary for in vitro chondrogenesis in elastin-like polypeptide biopolymer scaffolds. Tissue Eng Part A 2010;16:11-20.

[51] O'Halloran DM, Pandit AS. Tissue-engineering approach to regenerating the intervertebral disc. Tissue Eng 2007;13:1927-54.

[52] Bowles RD, Gebhard HH, Hartl R, Bonassar LJ. Tissue-engineered intervertebral discs produce new matrix, maintain disc height, and restore biomechanical function to the rodent spine. Proc Natl Acad Sci U S A 2011;108:13106-11.

[53] Nerurkar NL, Elliott DM, Mauck RL. Mechanics of oriented electrospun nanofibrous scaffolds for annulus fibrosus tissue engineering. J Orthop Res 2007;25:1018-28.

[54] Lutolf M, Hubbell J. Synthetic biomaterials as instructive extracellular microenvironments for morphogenesis in tissue engineering. Nat Biotechno 2005;23:47-55.

[55] Chiba K, Andersson GB, Masuda K, Thonar EJ. Metabolism of the extracellular matrix formed by intervertebral disc cells cultured in alginate. Spine 1997;22: 2885-93.

[56] Chou AI, Nicoll SB. Characterization of photocrosslinked alginate hydrogels for nucleus pulposus cell encapsulation. J Biomed Mater Res A 2009;91:187-94

[57] Wang B, Wu Y, Shao Z, Yang S, Che B, Sun C, et al. Functionalized selfassembling peptide nanofiber hydrogel as a scaffold for rabbit nucleus pulposus cells. J Biomed Mater Res A 2012;100:646-53.

[58] Bron JL, Helder MN, Meisel HJ, Van Royen BJ, Smit TH. Repair, regenerative and supportive therapies of the annulus fibrosus: achievements and challenges. Eur Spine J 2009;18:301-13.

[59] Leo BM, Li X, Balian G, Anderson DG. In vivo bioluminescent imaging of virusmediated gene transfer and transduced cell transplantation in the intervertebral disc. Spine 2004;29:838-44.

[60] Troy T, Jekic-McMullen D, Sambucetti L, Rice B. Quantitative comparison of the sensitivity of detection of fluorescent and bioluminescent reporters in animal models. Mol Imaging 2004;3:9-23. 